\title{
A combined dark matter study of AMS-02 antiprotons and Fermi-LAT gamma rays
}

\author{
Alessandro Cuoco, Jan Heisig*, Michael Korsmeier and Michael Krämer \\ Institute for Theoretical Particle Physics and Cosmology, RWTH Aachen University, 52056 \\ Aachen, Germany \\ E-mail: cuoco@physik.rwth-aachen.de, heisig@physik.rwth-aachen.de, \\ korsmeieraphysik.rwth-aachen.de, mkraemerephysik.rwth-aachen.de
}

Observations of cosmic rays are a sensitive probe of dark matter annihilation in our Galaxy. In this article we present an analysis of the AMS-02 antiproton data, reducing cosmic-ray propagation uncertainties by fitting at the same time dark matter and propagation parameters. The result exhibits a possible hint for dark matter pointing to an annihilation cross section close to the thermal value. We investigate the compatibility of this signal with a dark matter interpretation of the Galactic center excess seen in the Fermi-LAT gamma-ray data and discuss implications for dark matter models.

EPS-HEP 2017, European Physical Society conference on High Energy Physics 5-12 July 2017

Venice, Italy

${ }^{*}$ Speaker. 


\section{Introduction}

The nature of dark matter (DM) in the Universe constitutes one of the most pressing questions in contemporary fundamental physics. It has motivated an impressive joint effort in the field of particle- and astrophysics. Among the various strategies, indirect detection searches are of particular importance as they probe the self-annihilating nature of DM, potentially establishing a thermal freeze-out scenario as an explanation of the measured DM relic density. With the recently published data of the AMS-02 experiment on-board the international space station the cosmic-ray (CR) precision era has begun, allowing for a significant improvement in the sensitivity of DM searches. However, DM constraints are largely affected by uncertainties in the description of CR propagation in the Galaxy. This results in an order-of-magnitude uncertainty in the DM interpretation of CR fluxes when derived for benchmark propagation models, like the MIN/MED/MAX scenarios [1].

Here, we summarize our recent work [2,3] where we exploit the precise AMS-02 measurements of the fluxes of protons, helium and antiprotons. By preforming a joint fit of the propagation parameters and DM signal we minimize the CR propagation uncertainties. This treatment explores possible correlations and degeneracies between CRs and DM, providing more robust and reliable DM constraints. While being able to put strong constraints on the annihilation cross section at large masses, we find a statistically significant (around $4.5 \sigma$ local) indication of a signal from DM annihilation for lower masses $m_{\mathrm{DM}} \lesssim 100 \mathrm{GeV}$ pointing to an annihilation cross section close to the thermal one, $\langle\sigma v\rangle \simeq 3 \times 10^{-26} \mathrm{~cm}^{3} \mathrm{~s}^{-1}$. We analyse this indication for a variety of annihilation channels. Intriguingly, this possible signal from DM is largely compatible with the Galactic center excess (GCE) seen in the Fermi-LAT gamma-ray data.

\section{Cosmic-ray analysis}

The propagation of charged CRs can be described by a diffusion equation [4] for the particle density $\psi_{i}$ of species $i$ per volume and absolute value of momentum $p$

$$
\begin{aligned}
\frac{\partial \psi_{i}(\boldsymbol{x}, p, t)}{\partial t}= & q_{i}(\boldsymbol{x}, p)+\boldsymbol{\nabla} \cdot\left(D_{x x} \boldsymbol{\nabla} \psi_{i}-\boldsymbol{V} \psi_{i}\right)+\frac{\partial}{\partial p} p^{2} D_{p p} \frac{\partial}{\partial p} \frac{1}{p^{2}} \psi_{i} \\
& -\frac{\partial}{\partial p}\left(\frac{\mathrm{d} p}{\mathrm{~d} t} \psi_{i}-\frac{p}{3}(\boldsymbol{\nabla} \cdot \boldsymbol{V}) \psi_{i}\right)-\frac{1}{\tau_{f, i}} \psi_{i}-\frac{1}{\tau_{r, i}} \psi_{i}
\end{aligned}
$$

where $q_{i}(\boldsymbol{x}, p)$ is the source term of primary CRs and the terms proportional to $D_{x x}, \boldsymbol{V}$, and $D_{p p}$ correspond to $\mathrm{CR}$ diffusion, convection and reacceleration, respectively. The second line of eq. (2.1) includes the momentum gain or loss rate $\propto \mathrm{d} p / \mathrm{d} t$, adiabatic energy losses $\propto \nabla \cdot V$, and the loss by fragmentation and radioactive decay $\propto 1 / \tau_{f, i}$ and $1 / \tau_{r, i}$, respectively. We solve eq. (2.1) numerically using GALPROP [5] approximating the Galaxy by a cylindrical volume assuming a steady state regime. The source term for primary astrophysical CRs is modeled by a double broken power law with smooth transitions. We allow for individual spectral indices for proton and helium. Diffusion is modeled by a power law in rigidity and convective winds are assumed to be orthogonal to the Galactic plane. The respective parametrizations are detailed in [6] and introduce a total of 11 background propagation parameters. 
The source term for primary antiprotons from DM annihilation can be written as:

$$
q_{\bar{p}}^{(\mathrm{DM})}\left(\boldsymbol{x}, E_{\mathrm{kin}}\right)=\frac{1}{2}\left(\frac{\rho(\boldsymbol{x})}{m_{\mathrm{DM}}}\right)^{2} \sum_{f}\langle\sigma v\rangle_{f} \frac{\mathrm{d} N_{\bar{p}}^{f}}{\mathrm{~d} E_{\mathrm{kin}}},
$$

where $m_{\mathrm{DM}}$ denotes the DM mass, $\rho(\boldsymbol{x})$ the DM density profile, $\langle\sigma v\rangle_{f}$ the thermally averaged annihilation cross section and $\mathrm{d} N_{\bar{p}}^{f} / \mathrm{d} E_{\text {kin }}$ the corresponding antiproton energy spectrum per DM annihilation, see $[2,3]$ for further details. The factor $1 / 2$ in eq. (2.2) corresponds to Majorana fermion DM, the corresponding factor for a Dirac fermion is $1 / 4$. For a given annihilation channel $f, \mathrm{DM}+\mathrm{DM} \rightarrow f+\bar{f}$, the source term introduces two additional parameters, $m_{\mathrm{DM}}$ and $\langle\sigma v\rangle_{f}$. The resulting 13 parameters are determined in a global fit of the AMS-02 proton and helium fluxes [7, 8] and the AMS-02 antiproton-to-proton ratio [9], supplemented by proton and helium data from CREAM [10] and VOYAGER [11]. We use MultiNest [12] for an efficient sampling. The VOYAGER data, directly measuring the local interstellar space flux, is used to constrain the solar modulation potential which we describe in the framework of the force-field approximation.

\section{Cosmic-ray results}

We are able to put strong constraints on the annihilation cross section at large DM masses, see figure 1 (left) for the $b \bar{b}$ annihilation channel. ${ }^{1}$ However, in the mass range around and below $100 \mathrm{GeV}$ the global fit of the CR antiproton data improves significantly when adding a contribution of primary antiprotons from DM annihilation. This is due to a sharp spectral feature in the antiproton flux at a rigidity of about $20 \mathrm{GV}$, which is well fitted by the spectrum from DM annihilation due to the kinematic cut-off set by the DM mass. The feature cannot be described well by the secondary antiprotons produced by the interactions of primary protons and helium nuclei on the interstellar medium, since they only provide a smooth spectrum.

Figure 1 (right) shows the best fit regions in the DM mass and annihilation cross section for the six considered annihilation channels. The regions are obtained by minimizing the $\chi^{2}$ with respect to the remaining 11 parameters in the fit. Hence, they include the uncertainties in the CR source spectra and CR propagation. All channels provide an improvement compared to a fit without DM. For the latter we obtain a $\chi^{2} /($ number of degrees of freedom) of $71 / 165$. This is reduced to

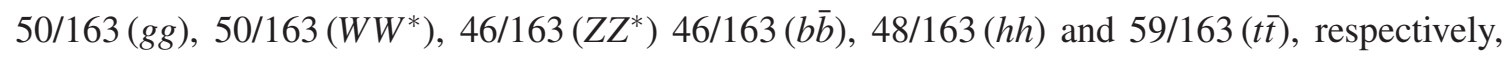
when adding a corresponding contribution from DM annihilation. Formally, $\Delta \chi^{2} \simeq 25$ (obtained for $b \bar{b}$ and $Z Z^{*}$ ) corresponds to a statistical significance of $4.5 \sigma .^{2}$ Figure 1 (right) also shows that different annihilation channels imply different preferred DM masses, ranging from $m_{\mathrm{DM}} \simeq 35 \mathrm{GeV}$ for gluons to $m_{\mathrm{DM}}$ near the Higgs or top mass for annihilation into $h h$ or $t \bar{t}$, respectively. Note that for the channels $W W^{*}$ and $Z Z^{*}$ the best fit masses lie below the corresponding on-shell production thresholds. We therefore take into account the respective 3-body final state processes via one offshell vector boson, denoted by the asterisk. For all channels the fit points to an annihilation cross section close to the value required for a thermal relic, $\langle\sigma v\rangle \simeq 3 \times 10^{-26} \mathrm{~cm}^{3} \mathrm{~s}^{-1}$.

A deeper scrutiny of this potential signal would require a more accurate determination of the antiproton production cross section, an accurate modeling of solar modulation at low rigidities

\footnotetext{
${ }^{1}$ For CR constraints on a variety of other annihilation channels, see [13].

${ }^{2}$ Note that this estimate does not account for possible systematic errors.
} 

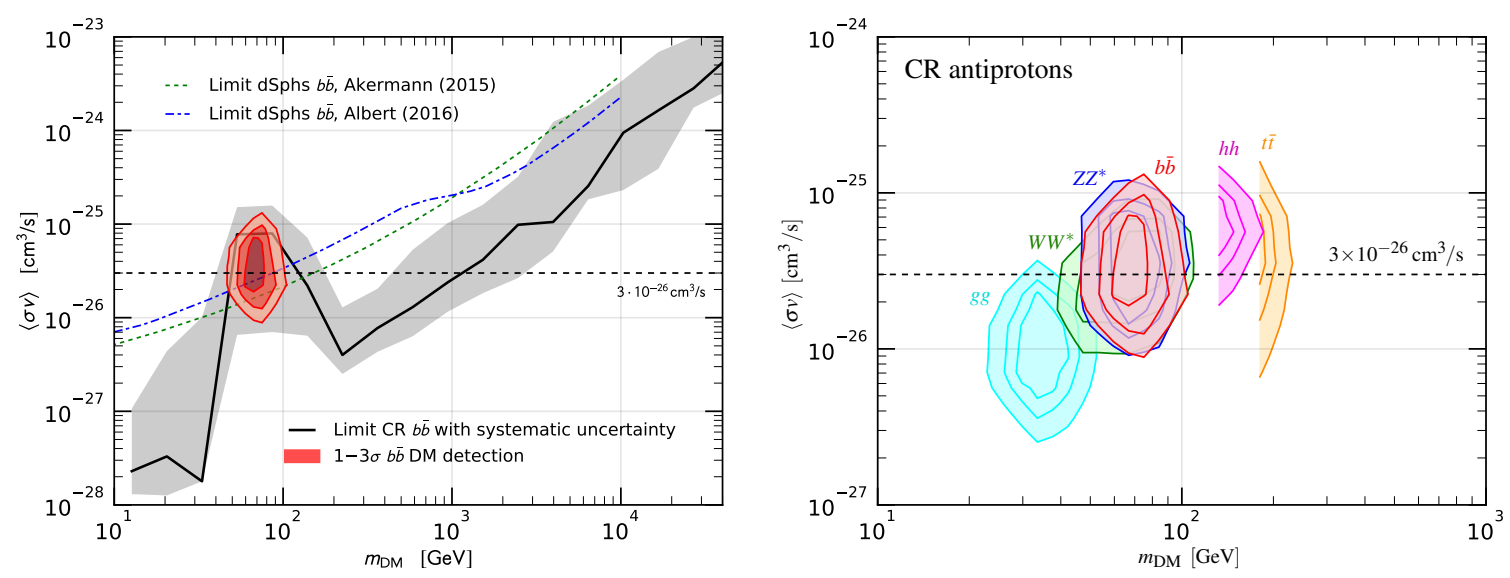

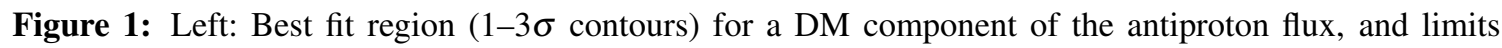
on the DM annihilation cross section into $b \bar{b}$ final states; from [2]. The grey shaded band denotes the systematic uncertainty. For comparison we show limits on the annihilation cross section obtained from gamma-ray observations of dwarf galaxies [14,15], and the thermal value of the annihilation cross section,

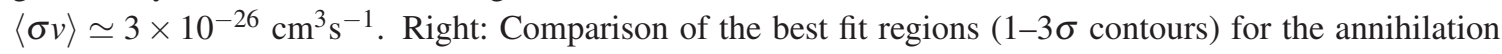
channels $g g$ (cyan), $W W^{*}$ (green), $b \bar{b}$ (red), $Z Z^{*}$ (blue), $h h$ (pink) and $t \bar{t}$ (orange); from [3].

of less than about $5 \mathrm{GV}$ as well as a better knowledge of correlations in the uncertainties of the AMS-02 data.

\section{Joint fit with Fermi-LAT gamma-ray data}

Annihilation of DM would also result in a continuum flux of gamma rays, predominantly from the decay of pions produced in the fragmentation of primary annihilation products. Accordingly, in this section we study whether the indication for a DM annihilation signal in CRs found in the last section is compatible with recent Fermi-LAT gamma-ray data. In particular, we consider data from dwarf spheroidal galaxies [15] and the so-called Galactic center excess (GCE) recently confirmed by the Fermi-LAT collaboration [16].

We consider a total of eleven dwarfs: the seven brightest confirmed dwarfs analyzed in [15] as well as Willman 1, Tucana III, Tucana IV and Indus II. We use the public likelihood provided by Fermi-LAT [15] and marginalize over the $J$-factors according to their uncertainties. For the GCE we use the gamma-ray energy spectrum and error covariance matrix obtained in [17]. We then perform a joint global fit using the gamma-ray and CR likelihoods (marginalized over CR propagation parameters). The gamma-ray fit follows the methodology described in [18]. Besides the two model parameters, $\langle\sigma v\rangle$ and $m_{\mathrm{DM}}$, the fit introduces two nuisance parameters - the $J$ factor for the Galactic center and the local DM density affecting the DM antiproton flux, which are varied around $\log \left(J_{\mathrm{GC}} /\left(\mathrm{GeV}^{2} \mathrm{~cm}^{-5}\right)\right)=53.54 \pm 0.43$ [18] and $\rho_{\odot}=0.43 \pm 0.15 \mathrm{GeV} / \mathrm{cm}^{3}$ [19], respectively. These two parameters take into account the uncertainty in the DM density profile and can approximately be considered to be independent [3].

Figure 2 (left) shows the preferred range of DM masses and annihilation cross sections, where we have marginalized over $J_{\mathrm{GC}}$ and $\rho_{\odot}$. For most annihilation channels, we observe very good agreement between the best fit regions of the CR signal and the DM interpretation of the GCE 

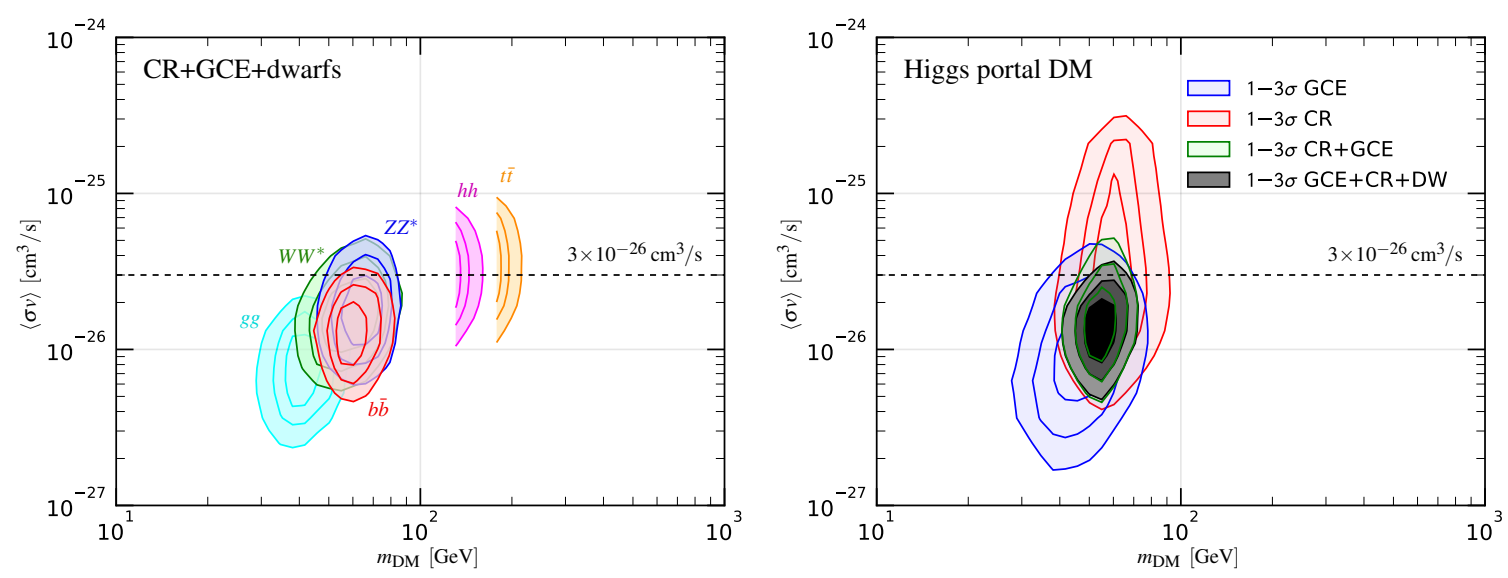

Figure 2: Left: Same as figure 1 (right) but taking into account the likelihood of Fermi-LAT dwarfs and the GCE in addition. Right: Individual and joint fits within the Higgs portal DM model; from [3].

while both are in accordance with limits from dwarf spheroidal galaxies. However, annihilation into gluons or top quarks is disfavored as an explanation of both the CR antiproton flux and the GCE. While both measurements individually prefer different regions of the DM mass for annihilation into gluons, annihilation into $t \bar{t}$ does not provide a particularly good fit to both the GCE and the antiprotons.

\section{Implications for dark matter models}

Realistic DM models often feature an admixture of various annihilation channels. It is thus not clear $a$ priori if a certain combination of annihilation channels, considered individually in the last sections, can provide a good fit of the observations. Here we consider the Higgs portal model where a DM field $X$ is directly coupled to the Higgs bilinear through the operator $X^{2} H^{\dagger} H$. Independent of the nature of $X$, below the Higgs-pair production threshold, the relative weights of the annihilation channels are solely determined by the DM mass. Accordingly, we can provide a joint fit of the CR and gamma-ray data within the Higgs portal model in terms of the two parameters $m_{\mathrm{DM}}$ and $\langle\sigma v\rangle$. The result is shown in figure 2 (right), where we have, again, marginalized over $J_{\mathrm{GC}}$ and $\rho_{\odot}$. The preferred region of DM masses is around $m_{\mathrm{DM}} \simeq 60 \mathrm{GeV}$, where the model predicts annihilation into $b \bar{b}, W W^{*}$ and $g g$ with a weight of approximately $70 \%, 20 \%$, and $10 \%$, respectively. We find a $\chi_{\mathrm{CR}}^{2} /($ number of degrees of freedom) of $47 / 163$ (compared to $71 / 165$ without DM). Intriguingly the preferred mass of $m_{\mathrm{DM}} \simeq 60 \mathrm{GeV}$ in the fit coincides with the narrow mass region around half of the Higgs mass which allows to reconcile existing constraints from direct detection, the relic density and collider searches, see [3] for a detailed analysis and discussion.

\section{Conclusion}

With the recently released AMS-02 data the cosmic-ray precision era has started. In order to reduce uncertainties with respect to the MIN/MED/MAX propagation scenarios we performed a joint fit of propagation and DM parameters. Intriguingly we found a feature in the antiproton flux 
which could be a possible hint for DM annihilation in the Galaxy. We explore a wide class of annihilation channels including $g g, b \bar{b}, W W^{*}, Z Z^{*}, h h$ and $t \bar{t}$ which provide a statistical improvement of the fit at the $4 \sigma$ level ( $3 \sigma$ level for $t \bar{t}$ ) with masses ranging from $35 \mathrm{GeV}$ to the Higgsand top-production thresholds. A further investigation of these potential DM signals would have to examine systematic effects resulting from solar modulation, secondary antiproton production, and correlations in AMS-02 data.

Interestingly, for most channels the signal is largely compatible with a DM interpretation of the GCE as well as the constraints from dwarf spheroidal galaxies. As an example, we perform a joint fit for the specific case of the Higgs portal DM model which points to a DM mass near half of the Higgs mass - a region which is also favored by totally independent constraints, namely the interplay of the relic density and direct detection limits.

\section{References}

[1] F. Donato, N. Fornengo, D. Maurin and P. Salati, Antiprotons in cosmic rays from neutralino annihilation, Phys. Rev. D69 (2004) 063501, [astro-ph/ 0306207 ].

[2] A. Cuoco, M. Krämer and M. Korsmeier, Novel Dark Matter Constraints from Antiprotons in Light of AMS-02, Phys. Rev. Lett. 118 (2017) 191102, [1610.03071].

[3] A. Cuoco, J. Heisig, M. Korsmeier and M. Krämer, Probing dark matter annihilation in the Galaxy with antiprotons and gamma rays, 1704.08258.

[4] A. W. Strong, I. V. Moskalenko and V. S. Ptuskin, Cosmic-ray propagation and interactions in the Galaxy, Ann. Rev. Nucl. Part. Sci. 57 (2007) 285-327, [astro-ph/0701517].

[5] A. W. Strong, I. V. Moskalenko and O. Reimer, Diffuse continuum gamma-rays from the galaxy, Astrophys. J. 537 (2000) 763-784, [astro-ph/9811296].

[6] M. Korsmeier and A. Cuoco, Galactic cosmic-ray propagation in the light of AMS-02: Analysis of protons, helium, and antiprotons, Phys. Rev. D94 (2016) 123019, [1607.06093].

[7] AMS collaboration, M. Aguilar et al., Precision Measurement of the Proton Flux in Primary Cosmic Rays from Rigidity 1 GV to 1.8 TV with the Alpha Magnetic Spectrometer on the International Space Station, Phys. Rev. Lett. 114 (2015) 171103.

[8] AMS collaboration, M. Aguilar et al., Precision Measurement of the Helium Flux in Primary Cosmic Rays of Rigidities 1.9 GV to 3 TV with the Alpha Magnetic Spectrometer on the International Space Station, Phys. Rev. Lett. 115 (2015) 211101.

[9] AMS collaboration, M. Aguilar et al., Antiproton Flux, Antiproton-to-Proton Flux Ratio, and Properties of Elementary Particle Fluxes in Primary Cosmic Rays Measured with the Alpha Magnetic Spectrometer on the International Space Station, Phys. Rev. Lett. 117 (2016) .

[10] Y. S. Yoon et al., Cosmic-Ray Proton and Helium Spectra from the First CREAM Flight, Astrophys. J. 728 (2011) 122, [1102.2575].

[11] E. C. Stone et al., Voyager 1 Observes Low-Energy Galactic Cosmic Rays in a Region Depleted of Heliospheric Ions, Science 341 (2013) .

[12] F. Feroz, M. P. Hobson and M. Bridges, MultiNest: an efficient and robust Bayesian inference tool for cosmology and particle physics, Mon. Not. Roy. Astron. Soc. 398 (2009) 1601-1614, [080 9 . 3437].

[13] A. Cuoco, J. Heisig, M. Korsmeier and M. Krämer, in preparation, 2017. 
[14] FERMI-LAT collaboration, M. Ackermann et al., Searching for Dark Matter Annihilation from Milky Way Dwarf Spheroidal Galaxies with Six Years of Fermi Large Area Telescope Data, Phys. Rev. Lett. 115 (2015) 231301, [1503.02641].

[15] DES, FERMI-LAT collaboration, A. Albert et al., Searching for Dark Matter Annihilation in Recently Discovered Milky Way Satellites with Fermi-LAT, Astrophys. J. 834 (2017) 110, [1611. 03184 ].

[16] FERMI-LAT collaboration, M. Ajello et al., Fermi-LAT Observations of High-Energy $\gamma$-Ray Emission Toward the Galactic Center, Astrophys. J. 819 (2016) 44, [1511.02938].

[17] F. Calore, I. Cholis and C. Weniger, Background model systematics for the Fermi GeV excess, JCAP 1503 (2015) 038, [1409.0042].

[18] A. Cuoco, B. Eiteneuer, J. Heisig and M. Krämer, A global fit of the $\gamma$-ray galactic center excess within the scalar singlet Higgs portal model, JCAP 1606 (2016) 050, [1603 . 08228].

[19] P. Salucci, F. Nesti, G. Gentile and C. F. Martins, The dark matter density at the Sun's location, Astron. Astrophys. 523 (2010) A83, [1003.3101]. 